\title{
Post-Intensive Care Unit Psychiatric Comorbidity and Quality of Life
}

\author{
Sophia Wang, MD ${ }^{1,2,3,4 *}$ and Chris Mosher, MD5, Anthony J. Perkins, MS,6, Sujuan Gao, PhD7, Sue Lasiter, RN, PhD², \\ Sikandar Khan, MD9 , Malaz Boustani, MD, MPH ${ }^{4,10,11}$, Babar Khan, MD, MS ${ }^{9,11}$
}

\begin{abstract}
${ }^{1}$ Department of Psychiatry, Indiana University School of Medicine, Indianapolis, Indiana; ${ }^{2}$ Roudebush VA Medical Center, Indianapolis, Indiana; ${ }^{3}$ Center of Health Innovation and Implementation Science, Center for Translational Science and Innovation, Indianapolis, Indiana; ${ }^{4}$ Sandra Eskenazi Center for Brain Care Innovation, Eskenazi Hospital, Indianapolis, Indiana; ${ }^{5}$ Department of Internal Medicine, Indiana University School of Medicine, Indianapolis, Indiana; ${ }^{6}$ Indiana Clinical and Translational Research Institute, Indiana University School of Medicine, Indianapolis, Indiana; ${ }^{7}$ Depart-

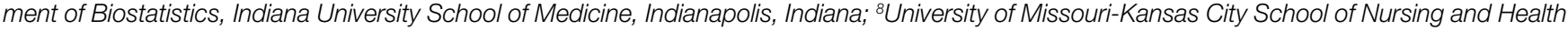
Studies, Kansas City, Missouri; 'Division of Pulmonary, Critical Care, Sleep and Occupational Medicine, Department of Internal Medicine, Indiana University School of Medicine, Indianapolis, Indiana; ${ }^{10}$ Division of Geriatrics and General Internal Medicine, Department of Internal Medicine, Indiana University School of Medicine, Indianapolis, Indiana; ${ }^{11}$ Indiana University Center of Aging Research, Regenstrief Institute, Indianapolis, Indiana.
\end{abstract}

The prevalence of psychiatric symptoms ranges from $17 \%$ to $44 \%$ in intensive care unit (ICU) survivors. The relationship between the comorbidity of psychiatric symptoms and quality of life (QoL) in ICU survivors has not been carefully examined. This study examined the relationship between psychiatric comorbidities and QoL in 58 survivors of ICU delirium. Patients completed 3 psychiatric screens at 3 months after discharge from the hospital, including the $\mathrm{Pa}-$ tient Health Questionnaire-9 (PHQ-9) for depression, the Generalized Anxiety Disorder-7 (GAD-7) questionnaire for anxiety, and the Post-Traumatic Stress Syndrome (PTSS10) questionnaire for posttraumatic stress disorder. $\mathrm{Pa}$ tients with 3 positive screens (PHQ-9 $\geq 10$; GAD-7 $\geq 10$; and PTSS-10 > 35) comprised the high psychiatric comorbidity group. Patients with 1 to 2 positive screens were labeled the low to moderate (low-moderate) psychiatric comorbidity group. Patients with 3 negative screens were labeled the no psychiatric morbidity group. Thirty-one percent of patients met the criteria for high psychiatric comorbidity. After adjusting for age, gender, Charlson Comorbidity Index, discharge status, and prior history of depression and anxiety, patients who had high psychiatric comorbidity were more likely to have a poorer QoL compared with the low-moderate comorbidity and no morbidity groups, as measured by a lower EuroQol 5 dimensions questionnaire 3-level Index (no, $0.69 \pm 0.25$; low-moderate, $0.70 \pm 0.19$; high, $0.48 \pm 0.24$; $P=0.017)$. Future studies should confirm these findings and examine whether survivors of ICU delirium with high psychiatric comorbidity have different treatment needs from survivors with lower psychiatric comorbidity. Journal of Hospital Medicine 2017;12:831-835. Published online first September 6, 2017. @ 2017 Society of Hospital Medicine
The prevalence of depression, anxiety, and posttraumatic stress disorder (PTSD) symptoms in intensive care unit (ICU) survivors ranges from $17 \%$ to $44 \%$. $^{1-4}$ Psychiatric comorbidity, the presence of 2 or more psychiatric disorders, is highly prevalent in survivors of acute respiratory distress syndrome and is associated with higher mortality in postsurgical ICU survivors. ${ }^{5-7}$ While long-term cognitive impairment in patients with ICU delirium has been associated with poor quality of life $(\mathrm{QoL}),{ }^{1}$ the effects of psychiatric comorbidity on QoL among similar patients are not as well understood. In this study, we examined whether psychiatric comorbidity was associated with poorer QoL in survivors of ICU delirium.

\section{METHODS}

We examined subjects who participated in the Pharmacologic Management of Delirium (PMD) clinical trial. This

*Address for correspondence and reprint requests: Sophia Wang, MD, 355 W 16th Street, Suite 4800 GH, Room 4250, Indianapolis, IN 46202; Telephone: 317-963-7288; Fax: 317-963-7313; E-mail: sophwang@iupui.edu

Received: November 29, 2016; Revised: March 28, 2017;

Accepted: April 4, 2017

2017 Society of Hospital Medicine DOI 10.12788/jhm.2827 trial examined the efficacy of a pharmacological intervention for patients who developed ICU delirium at a local tertiary-care academic hospital. ${ }^{8}$ Out of 62 patients who participated in the follow-up of the PMD study, 58 completed QoL interviews and validated psychiatric screens (Patient Health Questionnaire-9 [PHQ-9] for depression, the Generalized Anxiety Disorder-7 [GAD-7] questionnaire for anxiety, and the Post-Traumatic Stress Syndrome [PTSS-10] questionnaire for PTSD) at 3 months after hospital discharge. High psychiatric comorbidity was defined as having significant symptoms for all 3 conditions (depression: PHQ-9 score $\geq$ 10; anxiety: GAD $-7 \geq 10$; and PTSD: PTSS $-10>35)$. No psychiatric morbidity was defined as having no significant symptoms for all 3 conditions. Low to moderate (low-moderate) psychiatric morbidity was defined as having symptoms for 1 to 2 conditions.

Participants also completed 2 complementary QoL measures: the EuroQol 5 dimensions questionnaire 3-level (EQ5D-3L) Index and the EuroQol 5 dimensions Visual Analog Scale (EQ-5D-VAS). ${ }^{9}$ The EQ-5D-3L Index asks participants to rate themselves as having (1) no problems, (2) some problems, or (3) extreme problems on the following 5 scales: mobility, self-care, usual activities, pain/discomfort, and 
TABLE. Demographics and Clinical Characteristics of 58 ICU Survivors Grouped by Comorbidity of Psychiatric Disorder Symptoms

\begin{tabular}{|c|c|c|c|c|}
\hline Characteristics & $\begin{array}{c}\text { No Psychiatric } \\
\text { Morbidity } \\
(n=26)\end{array}$ & $\begin{array}{l}\text { Low to Moderate } \\
\text { Psychiatric } \\
\text { Comorbidity } \\
(n=14)\end{array}$ & $\begin{array}{l}\text { High Psychiatric } \\
\text { Comorbidity } \\
(n=18)\end{array}$ & $P$ Value \\
\hline \multicolumn{5}{|l|}{ Demographics } \\
\hline Age, years & $59.0(14.9)$ & $52.7(17.1)$ & $52.9(14.5)$ & .327 \\
\hline Female, \% (n) & $50.0(13)$ & $64.3(9)$ & $50.0(9)$ & .699 \\
\hline African American, \% (n) & $50.0(13)$ & $35.7(5)$ & $38.9(7)$ & .659 \\
\hline Education, years & $12.3(2.1)$ & $11.0(1.9)$ & $11.8(2.4)$ & .211 \\
\hline Prior depression \% (n) & $7.7(2)$ & $14.3(2)$ & $55.6(10)$ & $.001^{1, \mathrm{~b}}$ \\
\hline Prior anxiety, \% (n) & $3.8(1)$ & $0.0(0)$ & $22.2(4)$ & .081 \\
\hline Prior, PTSD, \% (n) & $0.0(0)$ & $0.0(0)$ & $0.0(0)$ & .000 \\
\hline IQCODE, mean (SD) & $3.1(0.2)$ & $3.1(0.2)$ & $3.1(0.1)$ & .929 \\
\hline \multicolumn{5}{|l|}{ Hospital Characteristics } \\
\hline $\begin{array}{l}\text { Service } \\
\text { MICU, \% } \\
\text { SICU, \% }\end{array}$ & $\begin{array}{c}73.1(19) \\
26.9(7)\end{array}$ & $\begin{array}{l}42.9(6) \\
57.1(8) \\
\end{array}$ & $\begin{array}{c}77.8(14) \\
22.2(4)\end{array}$ & .087 \\
\hline APACHE II score, mean (SD) & $18.6(9.1)$ & $18.9(9.0)$ & $20.5(8.1)$ & .768 \\
\hline ARF/Sepsis, \% (n) & $42.3(11)$ & $21.4(3)$ & $55.6(10)$ & .182 \\
\hline Coma, \% (n) & $76.9(20)$ & $92.9(13)$ & $88.9(16)$ & .460 \\
\hline Duration on ventilation, days (SD) & $6.7(7.4)$ & $7.6(4.8)$ & $8.7(9.9)$ & .701 \\
\hline Length of ICU stay, mean days (SD) & $23.6(19.5)$ & $20.7(17.9)$ & $17.1(11.2)$ & .350 \\
\hline Benzodiazapine drip, \% (n) & $38.5(10)$ & $42.9(6)$ & $55.6(10)$ & .590 \\
\hline Dexmetatomidine drip, \% (n) & $15.4(4)$ & $35.7(5)$ & $33.3(6)$ & .255 \\
\hline Propofol drip, \% (n) & $69.2(18)$ & $71.4(10)$ & $88.9(16)$ & .328 \\
\hline Discharged Home, \% (n) & $38.5(10)$ & $50.0(7)$ & $72.2(13)$ & .088 \\
\hline
\end{tabular}

${ }^{a}$ No psychiatric comorbidity group significantly different from the high psychiatric comorbidity group.

bow-moderate psychiatric comorbidity group significantly different from the high psychiatric comorbidity group.

NOTES: Continuous variables were expressed as average (SD). Dichotomous variables were expressed as \% (N). Fischer's exact test was used to compare dichotomous outcomes for the 3 groups. One-way ANOVA was used to compare continuous outcomes for the 3 groups.

Patients who had significant depressive, anxiety, or posttraumatic stress disorder (PHQ-9 score $\geq 10$, GAD-7 $\geq 10$, and PTSS-10 >35) symptoms were in the high comorbidity psychiatry group.

Patients who had no significant symptoms were in the no morbidity psychiatry group. Patients who met criteria for 1-2 significant symptoms were in the low-moderate comorbidity psychiatry group. Abbreviations: ANOVA, analysis of variance; APACHE, Acute Physiologic and Chronic Health Evaluation; ARF, acute renal failure; GAD-7, Generalized Anxiety Disorder-7; ICU, intensive care unit; IQCODE, Informant Questionnaire on Cognitive Decline; MICU, medical intensive care unit; PHQ-9, Patient Health Questionnaire-9; PTSD, post-traumatic stress disorder; PTSS-10, Post-Traumatic Stress Syndrome; SD, standard deviation; SICU, surgical intensive care unit.

anxiety/depression. The scores are then indexed against the US population to create a continuous index scale ranging from -0.11 to 1.00 . A score of 1 represents perfect health, 0 represents death, and negative values indicate a health state worse than death. The EQ-5D-VAS asks participants to draw a line on a visual scale from an anchor box to the point that represents their health state. The score ranges from 0 being the worst imaginable health state to 100 being the best imaginable health state. Demographic information, clinical characteristics, and prior history of depression, anxiety, and PTSD were obtained through PMD study records and clinical records. The Charlson Comorbidity Index, which measures chronic comorbidities, and Acute Physiology and Chronic Health Evaluation II, which estimates acute severity of illness within 24 hours of ICU admission, were calculated from patients' available clinical information.

Fisher's exact tests were used to compare dichotomous outcomes. Analysis of variance (ANOVA) was used to compare continuous outcomes across the 3 psychiatric groups. Analysis of covariance (ANCOVA) was used to determine whether psychiatric comorbidity in survivors of ICU delirium was associated with QoL measures. Models were adjusted 


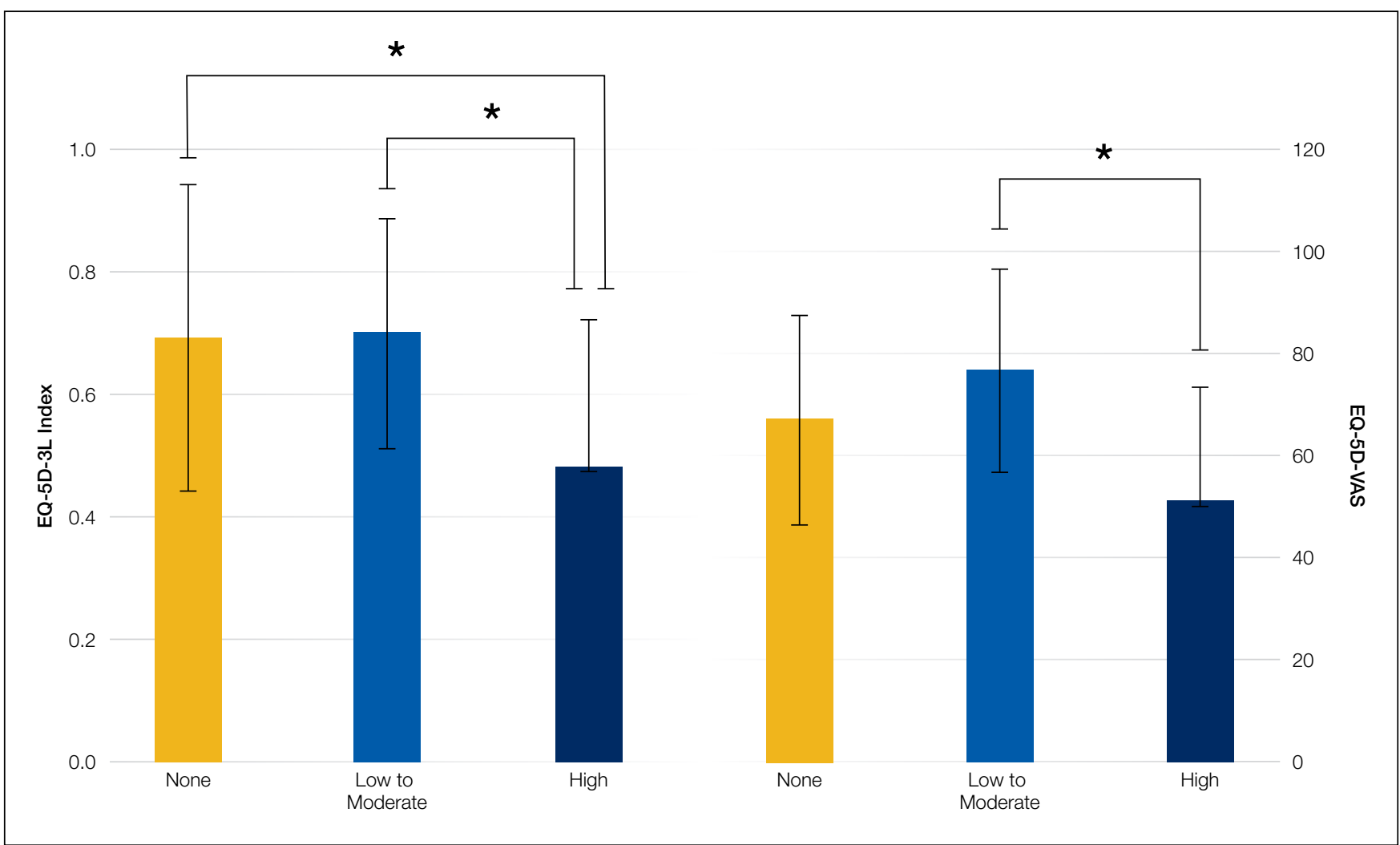

FIG. Mean EQ-5D-3L index or mean EQ-5D-VAS index grouped by psychiatric comorbidities. NOTE: ANCOVA models were adjusted for age, gender, Charlson Comorbidity Index, being discharged to home, prior history of depression, and prior history of anxiety. All $P$ values are after covariate adjustment. $P=.017$ for overall trend for EQ-5D-3L Index, and $P=.039$ for overall trend for EQ-5D-VAS. ${ }^{\star} P<.05$ for pairwise comparison. Abbreviations: ANCOVA, analysis of covariance; EQ-5D-3L, EuroQol 5 dimensions questionnaire 3-level; EQ-5D-VAS, EuroQol 5 dimensions Visual Analog Scale.

for the following covariates: age, gender, Charlson Comorbidity Index, discharged to home, prior history of depression, and prior history of anxiety. To assess the relationship of psychiatric comorbidity with QoL, we chose the 2 continuous QoL measures as the outcome. Because we were interested in the effect of psychiatric burden on QoL, we used ANCOVA with QoL as the dependent variable and psychiatric burden as an independent variable. Pairwise comparisons were then performed when overall differences were significant $(P$ $<0.05)$. We performed 2 separate sensitivity analyses. The first analysis looked solely at the subgroup of patients from the medical intensive care unit. We also recalculated the EQ-5D-3L index excluding the anxiety/depression item.

\section{RESULTS}

Nearly one-third of patients (18/58) had high psychiatric burden. The table looks at the demographic and clinical characteristics of patients with high psychiatric comorbidity versus those of low-moderate psychiatric comorbidity and those with no psychiatric morbidity. Patient groups did not differ significantly in terms of demographics. For clinical characteristics, patients with high psychiatric comorbidity were more likely than patients with low-moderate psychiatric comorbidity to have a prior history of depression $(P<0.05)$.

Patients with high psychiatric comorbidity were more like- ly to have a poorer QoL when compared with patients with low-moderate psychiatric comorbidity and to those with no morbidity as measured by a lower EQ-5D-3L Index (no, 0.69 \pm 0.25 ; low-moderate, $0.70 \pm 0.19$; high, $0.48 \pm 0.24 ; P=$ 0.006 ) and EQ-5D-VAS (no, $67.0 \pm 20.7$; low-moderate, $76.6 \pm 20.0$; high, $50.8 \pm 22.4 ; P=0.004)$. After adjusting for covariates, patients with high psychiatric comorbidity had a poorer QoL compared with those with no morbidity or low-moderate comorbidity on the EQ-5D-3L Index ( $P$ $=0.017$ for overall differences), whereas patients who had high psychiatric comorbidity had a poorer QoL compared to those with low-moderate comorbidity on the EQ-5D-VAS $(P=0.039$ for overall differences; Figure). Subgroup analysis of MICU patients yielded similar results. Patients with high psychiatric burden had significantly poorer QoL as measured by the EQ-5D-3L (unadjusted $P=0.044$, adjusted $P=0.003$ ) and the EQ-5D-VAS (unadjusted $P=0.007$, adjusted $P=$ $0.021)$. After excluding the anxiety/depression item from the EQ-5D-3L, we observed similar differences (no, $0.71 \pm$ 0.24 ; low-moderate, $0.75 \pm 0.15$; high, $0.58 \pm 0.22$; unadjusted $P=0.062$; adjusted $P=0.040$ ).

\section{DISCUSSION/CONCLUSION}

Psychiatric comorbidities in ICU survivors are common and pose a significant clinical issue. Patients with multiple psy- 
chiatric comorbidities can be more complicated to identify from a diagnostic standpoint and often require more prolonged, intensive mental health treatment when compared with patients with a single psychiatric disorder. ${ }^{10,11}$ Our study showed that high psychiatric comorbidity in survivors of ICU delirium is associated with a decreased QoL compared with those with no psychiatric comorbidity or with low-moderate psychiatric comorbidity. This finding is consistent with previous studies in the general population that patients with multiple psychiatric comorbidities are associated with a poorer QoL compared with patients with a single psychiatric comorbidity. ${ }^{10,11}$

There is a pressing need to better characterize psychiatric comorbidities in ICU survivors because our current evidence suggests that the prevalence of psychiatric comorbidities of ICU survivors is substantially higher than that of the general population. We found that nearly one-third of survivors of ICU delirium had comorbid depression, anxiety, and PTSD symptoms at 3 months. This is consistent with the few other studies of ICU survivors, which showed a prevalence of psychiatric comorbidity of $25 \%$ to $33 \%$. ${ }^{5,12}$ These rates are substantially higher than the prevalence in the general population of $6 \% .^{13}$

The high rate of psychiatric comorbidities may render it difficult to effectively treat the mental health symptoms in ICU survivors. ${ }^{14}$ Treating multiple psychiatric comorbidities may also be especially challenging in survivors of ICU delirium because they have a high prevalence of cognitive impairment. Mental health treatments for patients with psychiatric disorders and comorbid cognitive impairment are limited. Better characterization of psychiatric comorbidity in ICU survivors, particularly those with ICU delirium, is vital to the development of more effective, bundled treatments for this population with multiple comorbidities.

Standardized screenings of ICU survivors at a high risk for psychiatric disorders, such as survivors of ICU delirium, may help to identify patients with comorbid psychiatric disorder symptoms and have them referred to appropriate treatment earlier with the hope of improving their QoL sooner. Although opportunities to deliver integrated outpatient collaborative mental health and medical care for a subspecialty population are limited, one potential model of care would be to utilize a collaborative-care model in an ICU survivor clinic. ${ }^{15}$

Strengths of our study include the examination of psychiatric comorbidities in survivors of ICU delirium, who often have a poor QoL. A deeper understanding of psychiatric comorbidity and its relationship with QoL is needed to better understand how to deliver more effective treatments for these survivors. Limitations include the small sample size, a one-time measurement of psychiatric comorbidities at the 3-month follow-up based on screenings tools, and a lack of objective measures of physical functioning to determine the effects of psychiatric comorbidities on physical functioning. There may also have been differences in how patients with no psychiatric comorbidity responded to the EQ-5D-VAS as a result of premorbid differences (eg, they were healthier prior to their ICU stay and perceived their survivor status more negatively). This may explain why we did not see a statistically significant difference between no psychiatric comorbidity and high psychiatric comorbidity groups on the EQ-5D-VAS. Nevertheless, we did see a difference between the low-moderate psychiatric comorbidity group on EQ5D-VAS and differences between the no comorbidity and low-moderate comorbidity groups versus the high comorbidity group on the EQ-5D-3L. Finally, data about psychiatric history and QoL prior to ICU hospitalization were limited. Therefore, truly determining incidence versus prevalence of post-ICU comorbidities and whether psychiatric symptoms and its effects on QoL were due to ICU hospitalization or to premorbid psychiatric symptoms is difficult.

Our study demonstrated that in survivors of ICU delirium, higher comorbidity of psychiatric symptoms was associated with poorer QoL. Future studies will need to confirm these findings. We will also need to identify potentially reversible risk factors for psychiatric comorbidity and poorer QoL and develop treatments to effectively target the mental health symptoms of survivors of ICU delirium.

Disclosure: Grant support: The PMD trial is funded through the National Institutes of Health grant R01AG054205-02. SW is supported by NIA 2P30AG010133. AP is supported by CMS 1 L1 CMS331444-02-00, Indiana CTSI, and NIA R01AG054205-02. SG is supported by NIA 2P30AG010133, NIA 5R01AG045350, and NIA R01AG054205-02. SK is supported by NHBLI 5T32HL091816-07. MB is supported by NIA R01 AG040220-05, AHRQ P30 HS024384-02, CMS 1 L1 CMS331444-02-00, NIA R01 AG030618-05A1 and NIA R01AG054205-02. BK is supported by NIA K23-AG043476 and NHLBI R01HL131730. The funding agency had no role in the development of the study design, collection, analysis, interpretation of data, manuscript development, or the decision to submit the manuscript for publication. Conflicts of interest include MB, SG, and AP being funded by NIA R01AG054205-02 for the PMD study.

\section{References}

1. Jutte JE, Erb CT, Jackson JC. Physical, cognitive, and psychological disability following critical illness: what is the risk? Semin Respir Crit Care Med. 2015;36(6):943-958.

2. Nikayin S, Rabiee A, Hashem MD, et al. Anxiety symptoms in survivors of critical illness: a systematic review and meta-analysis. Gen Hosp Psychiatry. 2016;43: 23-29.

3. Rabiee A, Nikayin S, Hashem MD, et al. Depressive symptoms after critical illness: a systematic review and meta-analysis. Crit Care Med. 2016;44(9): 1744-1753.

4. Parker AM, Sricharoenchai T, Raparla S, Schneck KW, Bienvenu OJ, Needham DM. Posttraumatic stress disorder in critical illness survivors: a metaanalysis. Crit Care Med. 2015;43(5):1121-1129.

5. Bienvenu OJ, Colantuoni E, Mendez-Tellez PA, et al. Cooccurrence of and remission from general anxiety, depression, and posttraumatic stress disorder symptoms after acute lung injury: a 2-year longitudinal study. Crit Care Med. 2015;43(3): $642-653$

6. Huang M, Parker AM, Bienvenu OJ, et al. Psychiatric Symptoms in Acute Respiratory Distress Syndrome Survivors: A 1-Year National Multicenter Study. Crit Care Med. 2016;44(5):954-965.

7. Abrams TE, Vaughan-Sarrazin M, Rosenthal GE. Influence of psychiatric comorbidity on surgical mortality. Arch Surg. 2010;145(10):947-953.

8. Campbell NL, Khan BA, Farber M, et al. Improving delirium care in the intensive care unit: the design of a pragmatic study. Trials. 2011;12:139.

9. EuroQol Group. EuroQol--a new facility for the measurement of health-related quality of life. Health Policy. 1990;16(3):199-208.

10. Hirschfeld RM. The comorbidity of major depression and anxiety disorders: rec- 
ognition and management in primary care. Prim Care Companion J Clin Psychiatry. 2001;3(6):244-254

11. Campbell DG, Felker BL, Liu CF, et al. Prevalence of depression-PTSD comorbidity: implications for clinical practice guidelines and primary care-based interventions. J Gen Intern Med. 2007;22(6):711-718.

12. Wolters AE, Peelen LM, Welling MC, et al. Long-term mental health problems after delirium in the ICU. Crit Care Med. 2016;44(10):1808-1813.
13. Kessler RC, Chiu WT, Demler O, Merikangas KR, Walters EE. Prevalence, severity, and comorbidity of 12-month DSM-IV disorders in the National Comorbidity Survey Replication. Arch Gen Psychiatry. 2005;62(6):617-627.

14. Mehlhorn J, Freytag A, Schmidt K, et al. Rehabilitation interventions for postintensive care syndrome: a systematic review. Crit Care Med. 2014;42(5):1263-1271.

15. Khan BA, Lasiter S, Boustani MA. CE: critical care recovery center: an innovative collaborative care model for ICU survivors. Am J Nurs. 2015;115(3):24-31. 